\title{
Development of an electrostatic positioner for space material processing
}

\author{
W. K. Rhim, M. Collender, M. T. Hyson, W. T. Simms, and D. D. Elleman \\ Jet Propulsion Laboratory, California Institute of Technology, Pasadena, California 91109 \\ (Received 6 February 1984; accepted for publication 26 September 1984)
}

This paper describes an electrostatic positioning instrument which was developed at the Jet Propulsion Laboratory to enable experimenters to conduct containerless material science experiments in space. Samples that are to be studied are electrically charged and controlled by the electrostatic force produced by a set of properly arranged electrodes. Three different types of positioners are described, i.e., the dish type, the ring type, and the tetrahedral type. In all these systems, the positioning and the damping of the sample is accomplished by a feedback control system. The advantage of this electrostatic positioning method, in comparison to the other methods, such as acoustic and electromagnetic, lies in the fact that it can operate in a high vacuum and does not require the material to be electrically conductive as long as the material can carry a certain amount of charge.

\section{INTRODUCTION}

The space shuttle flights provide scientists with a unique environment of extended microgravity, in which they can conduct various experiments which will enhance their understanding of natural phenomena unperturbed by the strong gravitational field effects ever present in groundbased laboratories. One clear advantage of going to space for material science experimentation is that one can position, control, and manipulate large experimental objects with very weak control forces. ${ }^{1}$ Molten samples, for instance, can be positioned and controlled within a high-temperature container while preventing them from touching the container walls. Such a contactless approach will protect the sample specimen from undesirable contamination and reduce heterogeneous nucleation and deformation caused by physical contact with the container.

This paper introduces an electrostatic positioning device developed for material science experiments in space. In this method, the material is electrically charged and controlled by the electrostatic force produced by a set of properly arranged electrodes. Since the electrostatic field cannot possess a three-dimensional potential minimum, as a direct result of Earnshaw's theorem, ${ }^{2}$ positioning must be accomplished by controlling the electric field through a feedback control system. ${ }^{3}$

There are other positioning methods being tested for space applications: acoustic positioning methods ${ }^{4-6}$ and electromagnetic methods. ${ }^{7-10}$ Acoustic methods require the presence of a gas medium to sustain the acoustic wave. While gas pressure as low as 2-10 Torr may suffice, the method cannot be used in a high vacuum, which may be required if purification of molten material by outgassing into a vacuum is used. Electromagnetic methods require the materials to be electric conductors, though not necessarily good ones in a microgravity environment, and thus exclude a range of nonconducting materials. Electrostatic positioning is complimentary to other methods and alleviates some of their shortcomings. It can operate in a high vacuum and does not require conducting materials if the materials used can carry a certain amount of charge. Therefore, electrostatic methods are potentially important for positioning.

In the following sections, we will describe the basic design principles, system components, and performance of different positioners in different $g$ environments. The paper concludes with a discussion of the shortcomings of the present electrostatic positioners and suggestions of possible future improvements.

\section{POSHTHONHG SYSTEMS}

Our efforts at the Yet Propulsion Laboratory have centered on the development of three electrostatic positioners: the dish type, the ring type, ${ }^{1}$ and the tetrahedral type of positioner, which are shown in Figs. 1, 2, and 3 respectively.

\section{A. Dish-\$ype positioner}

This is the earliest version of the positioners. It has simplicity in both mechanical design and the servo control system. However, it is only operable in a ground-based environment, and will not operate in space. This is because the generation of centering force depends on the presence of a

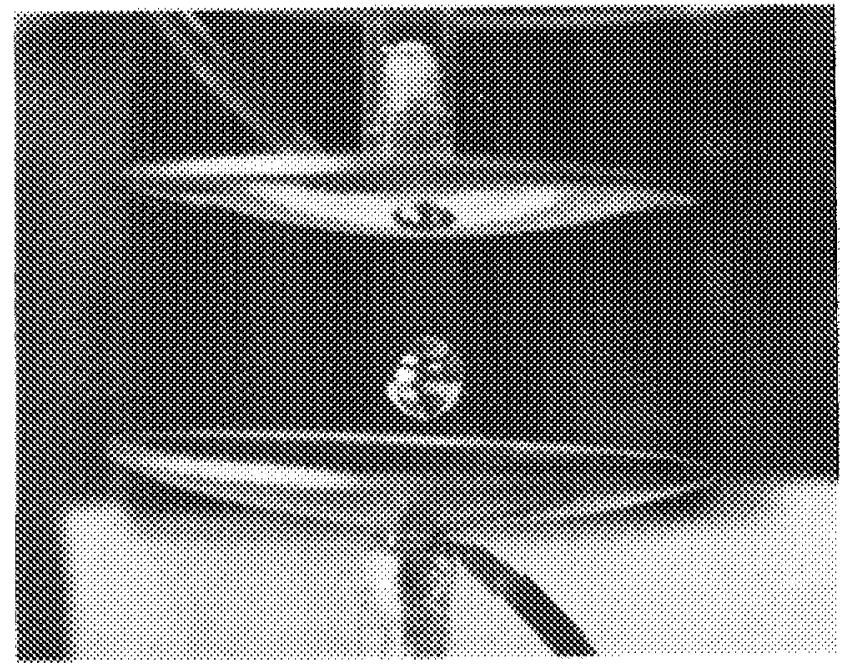

FIG. 1. A dish-type positioner. A platinum-coated glass shell is being levitated in a $1-g$ environment. The diameter of the shell is $\sim 0.5 \mathrm{in}$. and weight is $\sim 0.15 \mathrm{~g}$. 


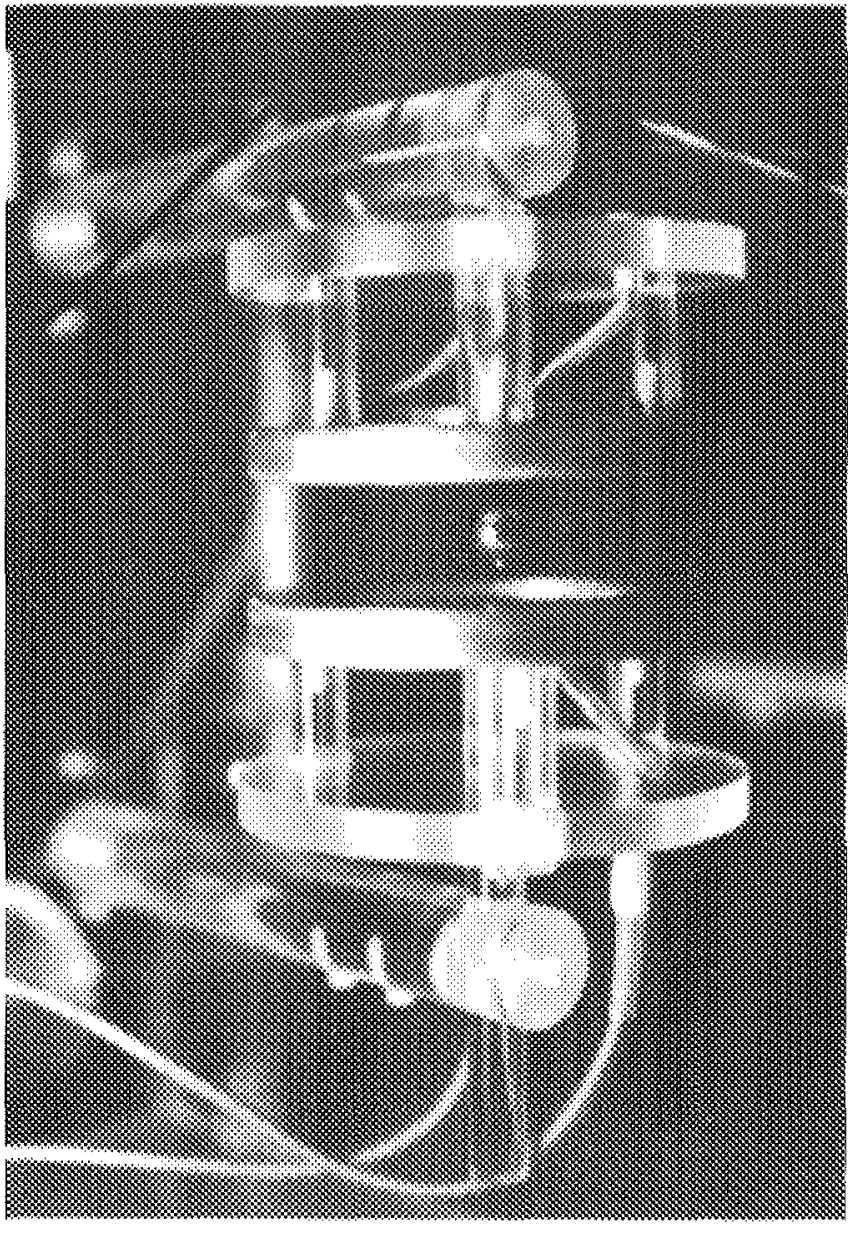

FIG. 2. A ring positioner which can be used in the various $g$ environments

large constant $g$ force. Assuming a pair of horizontal, parallel electrodes, the equation

$$
M g=(V / d) Q
$$

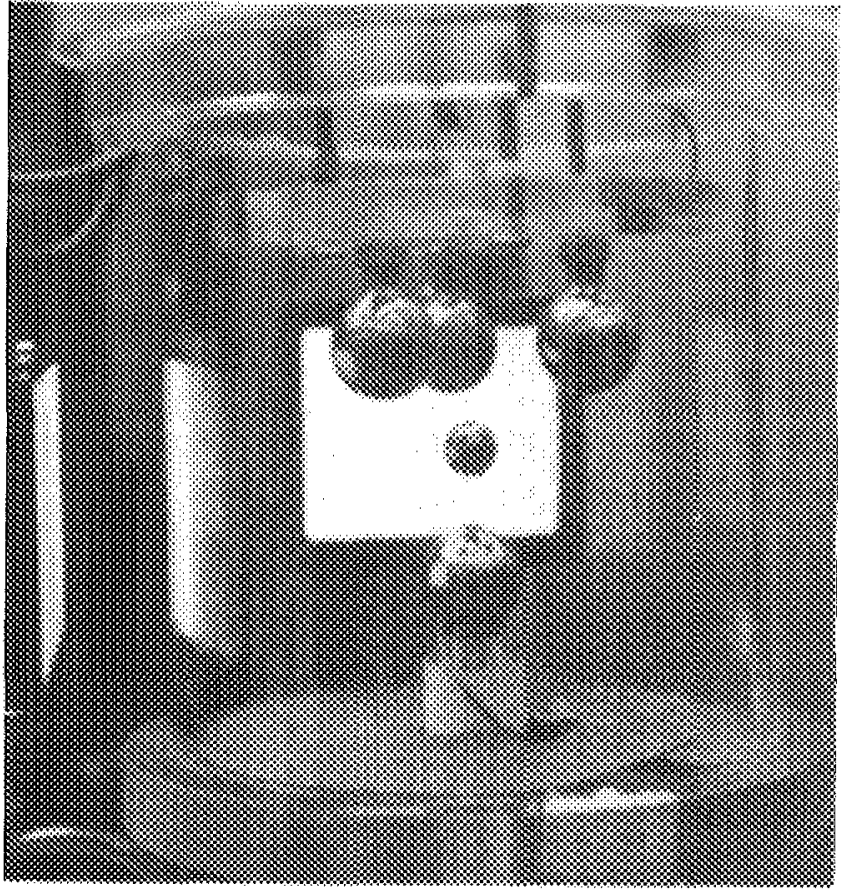

Fig. 3. A tetrahedral positioner. Three-dimensional positioning and damping have been achieved with this positioner in various $g$ environments. should be satisfied in order to levitate an object between the electrodes, where $M$ is the mass of the object carrying a charge $Q$, and $V$ is the voltage difference between the plate electrodes which are separated by a distance $d$. In practice, typical values of these parameters might be $Q=4 \times 10^{-9} \mathrm{C}$, $M=0.1 \mathrm{~g}, d \approx 1.2 \mathrm{~cm}$, and $V \approx 13 \mathrm{kV}$. If a pair of flat electrodes were used, there would be no laterally confining force. The confining force, however, can easily be generated by shaping the electrodes. In our design, we used a circular convex top electrode and a matching concave bottom electrode. The electrostatic lines of force may be decomposed into vertically lifting components and horizontally centering components. This centering force will increase as $(V Q / d R \mid X$, where $X$ is the distance of the object from the symmetry axis of the electrodes, and $R$ is the curvature radius of the electrodes. In a 1-g environment, the ever present large vertical force ensures a centering force of substantial strength. Since the system requires only a one-dimensional (uniaxial) control system, it is a simple and convenient system for groundbased operation.

\section{B. Ring positioner}

It was mentioned in the previous section that the dishtype positioner, though it is the simplest device for groundbased operation, cannot be operated in a zero-g environment. To be operable in zero-g, the positioner should be able to generate lateral centering forces at all levels of $g$. In order to do this, the ring positioner was developed.

As is in the dish-type positioner, on which the ring positioner is based, active positional control was possible only along the vertical symmetry axis. The ring positioner (see Fig. 4) uses and derives its name from the addition of two annular electrodes used to create a radially symmetric field to force a charged object into a two-dimensional potential well with respect to its movement in a lateral plane. This passively centers the object.

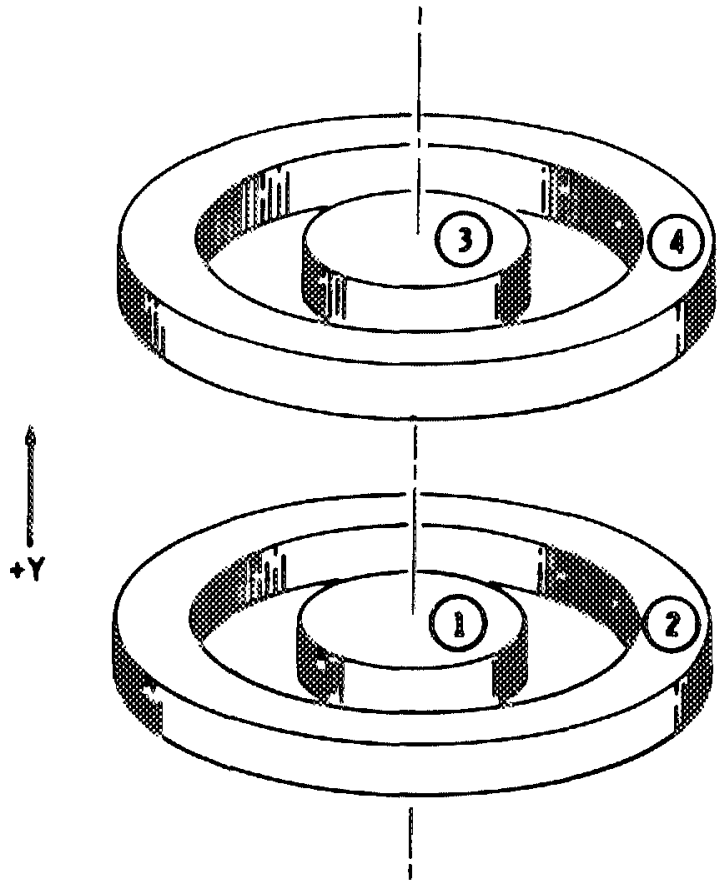

FIG. 4. The electrode arrangement of the ring positioner. 
As is evident in Fig. 4, the lateral centering force was produced by maintaining the ring electrodes 2 and 4 at higher voltage level than their respective central electrodes 1 and 3 at all times. In practice, the ring electrodes were maintained about $3.5 \mathrm{kV}$ higher than the central disk electrodes. The centering force this produced was estimated by measuring the period of an object's lateral oscillation which followed an initial displacement of its position and was found to be approximately $20 \mathrm{dyn}$.

\section{Tetrahedral posititioner}

Although the two positioners described above are easy to construct and operate, they are still devices in which active servo control can only be applied along their vertical symmetry axis. Therefore, along all other directions, the only damping force which can quiet the oscillatory motion of the sample around its potential minimum is the frictional force of the medium. No frictional forces will be present for an object levitated in a vacuum. The oscillation subjects the object to long-lasting periodic accelerations, defeating one purpose of space processing, which is to avoid acceleration.

The tetrahedral positioner resolves this problem by imposing active three-dimensional feedback control. It has four spherical electrodes in a tetrahedral arrangement $t^{12}$ and uses two solid-state charge coupled device (CCD) cameras to sense the three-dimensional coordinates of the object being levitated. Because an active feedback control system is used, the object can be positioned anywhere within the levitation space. Oscillations are actively damped by the feedback system in all directions. A density-one object will have its oscillations damped in less than $1 \mathrm{~s}$ as compared to acoustic positioners (which normally do not have feedback control) which take several minutes to damp similar oscillations using air drag alone. A detailed description of the system's components and control algorithms is presented below.

\section{SYSTEM COMPONENTS}

Despite differences described in the previous section, a single block diagram of the control electronics suffices for all positioners. Figure 5 shows the primary components of the

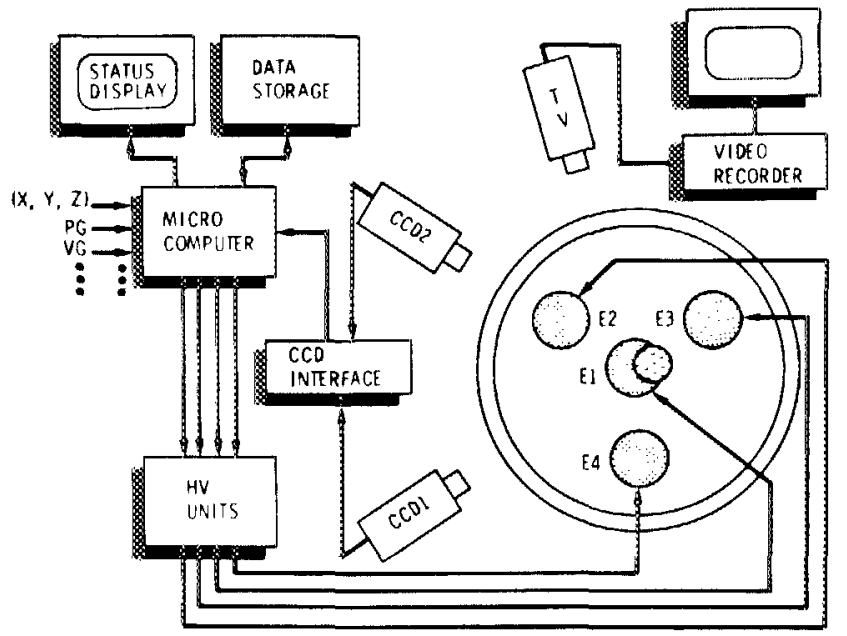

FIG. 5. A block diagram of the electrostatic positioning devices. This diagram applies for all positioners except for the number of electrodes and their arrangements. control system: (1) charge coupled device (CCD) cameras (EG\&G Reticon MC510D50X50-4, Sunnyvale, CA) for sensing the object's position, (2) interface logic to convert the video raster signal from the camera(s) to an 8-bit binary integer representing the object's position for each dimension of interest, (3) a microcomputer implementing the control algorithm, (4) four digital-to-analog converters which generate control voltages for (5) four programmable high-voltage units (see Sec. V and Fig. 11), and (6) a levitation chamber containing up to four electrodes. The object itself completes the levitation system. In addition, a cathode-ray tube monitor displays the status of the system and the keyboard interfaced to the control computer allows an operator to alter control parameters. A typical status display is shown in Fig. 6.

The CCD cameras were $50 \times 50$ pixel self-scan photodiode arrays generating a threshold light/dark image. Diffuse backlighting is used to make the levitated object appear as a dark group of pixels on a light background. The camera interface is a hardware device used to extract the centroid of the object's position from the two CCD camera images. The camera interface relieves the control computer of the image processing task which would otherwise consume most of its capacity.

The camera interface logic is triggered by a sync pulse from one of the CCD cameras at the start of a frame. Two registers hold the current horizontal and vertical pixel address of the ongoing CCD scan. If a pixel is above threshold, the column address is added to a column sum register and a total pixel sum register is incremented. At the end of a frame, the sum of the column addresses divided by the total number of pixels gives the average column position of the object. This is out put as the $x$-coordinate of the sample. The total number of pixels is divided by two and stored for use on the next frame. To obtain the $y$-coordinate, during the next frame, a counter is incremented for each pixel above threshold and compared to this stored value until its value is reached. Then, the row address when this occurs is output as the $y$ coordinate of the object.

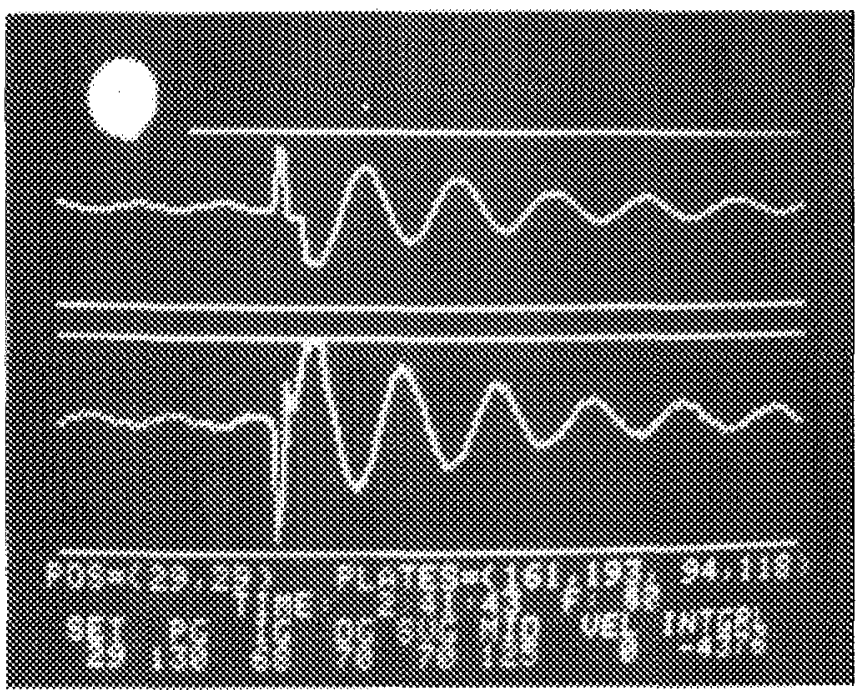

FIG. 6. A typical status display of the control system where SET = set position, $\mathbf{P G}=$ proportional gain, $I G=$ integral gain. $D G=$ derivative gain. $\mathrm{SVG}=$ slow velocity gain, and $\mathrm{MID}=$ midpoint . 
Thus, the $x$-coordinate of the object's centroid is measured as the average $x$-coordinate of all pixels above threshold and the $y$-coordinate is the current scan line when half the pixels which were above threshold on the previous frame have been counted.

For the dish or ring positioners, only the vertical (or $y$ ) axis is controlled and requires only one camera to measure the row position of the object's centroid. For full three-axis control used with the tetrahedral positioner, a second CCD camera is slaved to the first and placed orthogonally so that as the $z$ coordinate of the object changes, its horizontal position in the second CCD camera varies. The $z$ coordinate is computed in the same way as the $x$ coordinate already described.

On every start of frame, the camera interface issues an interrupt to the control computer and transmits 3 bytes of position data $(x, y, z)$ along a serial fiber-optic link to the control computer. A bit stream representing each camera's image is also transmitted. At the control computer, the position and image data are transferred by direct memory access (DMA) to the Apple's graphics memory page, where it is available to the servo program.

The camera's rate is 120 frames per second. The control computer receives updated coordinates of the object's centroid position and computes new control values with each frame.

A method for determining the object's position by find-

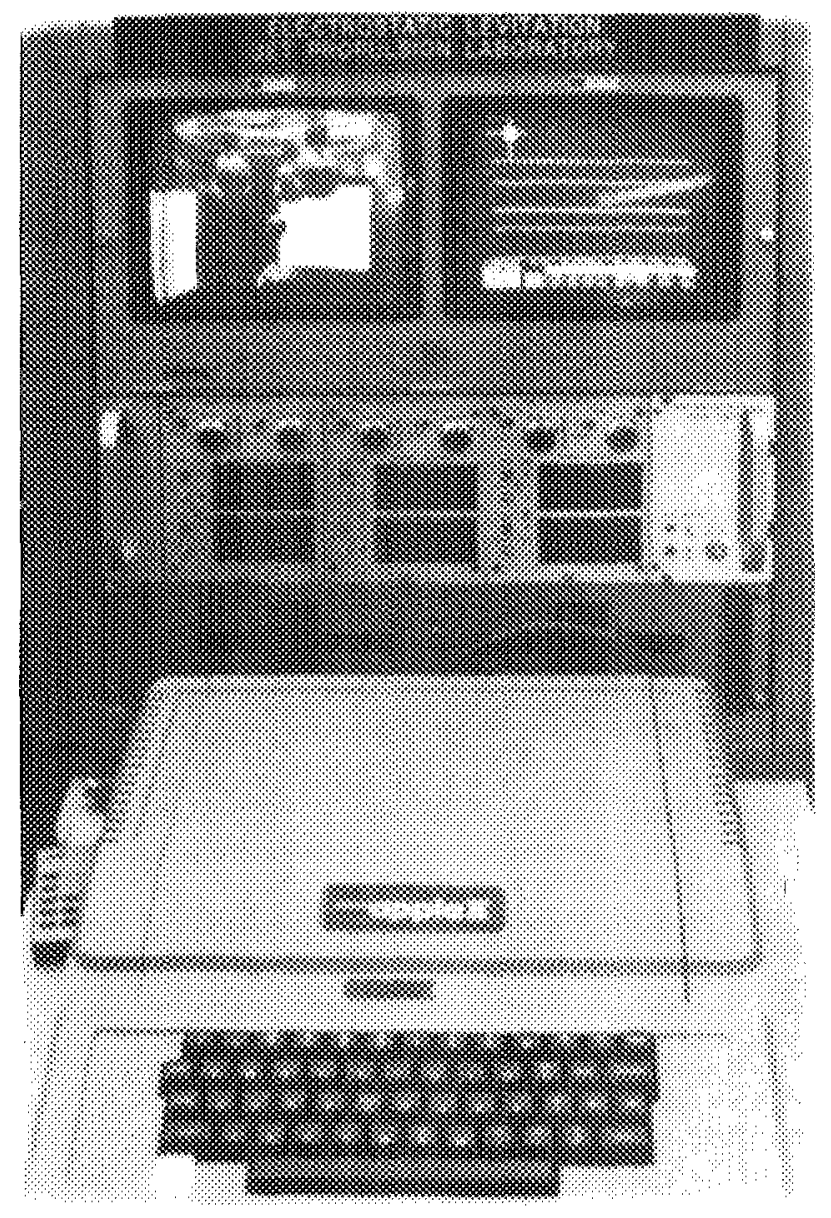

Fig. 7. A view of the control console. The units below the twin video monitors are the six high-voltage control units. ing the extrema of its image was simulated in software. On the first pass, the extrema of the object were found. This estimate was used to predict which area of the image to sample in the next frame. While the extrema finding method required fewer image samples, the above method was simpler to implement in the camera interface hardware.

Although it may be noted that the position measurements obtained with standard optics will not be in exact agreement with an ideal cubical grid because of perspective distortion, they are quite adequate for control purposes in this application. A view of the actual control console is shown in Fig. 7.

\section{UII. CONTROL ALGORITHMS}

The positioner apparatus, as implemented, may be properly described as a discrete-time, sampled-data, digital control system. In this case, the position of the levitated object is sampled at discrete intervals of $1 / 120$ th of a second and this measurement is used to compute 8-bit unsigned output values proportional to the controlling electrode voltages according to the algorithms described below.

The basic method of determining the outputs is the popular proportional-integral-derivative or PID servo algorithms for implementing a second-order linear feedback system. ${ }^{13}$ An error signal equal to the difference between the measured variable and its desired value, determine the output as follows:

$$
0(t)=M P+P G \cdot e(t)+I G \int_{o}^{t} e(\tau) d \tau+D G \frac{d e(t)}{d t},
$$

where

$e(t)=S P-y(t)=$ position error,

$S P=$ position setpoint in pixels,

$y(t)=$ controlled variable $=$ actual position in pixels,

$M P=$ midpoint $=$ value for integral offset (gravitational force compensation),

$$
\begin{aligned}
& P G=\text { proportional gain, } \\
& D G=\text { derivative gain, } \\
& I G=\text { integral gain. }
\end{aligned}
$$

In discrete form, Eq. (1) becomes

$$
\begin{aligned}
O(n)= & M P+P G \cdot E(n)+I G \sum_{i=0}^{n} E(i) \\
& +D G[E(n)-E(n-1)],
\end{aligned}
$$

where

$$
\begin{aligned}
& E(n)=S P-Y(n)=\text { error at } n \text {th sample time, } \\
& O(n)=\text { output at } n \text {th sample time, } \\
& Y(n)=\text { position, } n \text {th sample time, }
\end{aligned}
$$

$M P, S P, P G, I G, D G$, which are defined as above, are empirically determined to give stable servo behavior.

In the case of the actual levitation apparatus, it was found that a frame to frame difference was inadequate for the derivative, or velocity measurement, due to the limited $50 \times 50$ pixel resolution of the cameras and the high frame rate relative to velocities which a sample might typically assume. For the current apparatus and camera magnification, 
a 1-cm sample has an image size of about 20 pixels in diameter and usually moves one to five pixels per frame. Thus, the object's velocity ranges from 6 to $30 \mathrm{~cm} / \mathrm{s}$. Therefore, another term, called the slow velocity measurement, consisting of the difference between the latest position measurement and that from four frame-times before, was added to ensure adequate damping. The frame to frame difference was retained to provide rapid response to large perturbations. Our version of the PID servo is then

$$
\begin{aligned}
O(n)= & M P+P G \cdot E(n)+I G \sum_{i=0}^{n} E(i) \\
& +D G[E(n)-E(n-1)] \\
& +S V G[E(n)-E(n-4)],
\end{aligned}
$$

where $S V G=$ slow velocity gain. The midpoint value $(M P)$ is a positive offset (about 100 ) added to the output value. It compensates for the force of gravity during levitation in $1 \mathrm{~g}$. In principle, the integral term: $\sum_{O}^{n} E(i)$ would increase over time as the sample failed to reach its setpoint, until the output value $O(n)$ commanded sufficient voltage to lift the sample. In practice, the integral term is limited to a maximum value of \pm 32767 , because 2 bytes are used for storage. Addition of the MP term provides an initial force on the sample, lessens the chance that the integral term will overflow, and sets the servo at approximately the correct value at the time the sample is launched.

Equation (3), when implemented with binary integer arithmetic by a microcomputer with appropriate scaling factors, is adequate for controlling the dish-type positioner in a 1-g environment. Here the output of Eq. (3) is used to control the positive voltage on the lower electrode while the upper electrode is left at ground potential. The gain parameters were empirically adjusted for stability and dynamic response (see Fig. 1). Typical values used for the positioner in Fig. 1 were as follows: $M P=100 ; P G=80 ; I G=100 ; D G=75$; $S V G=20$ and $S P=20$ pixels (somewhat lower than midframe). These values yield an output $O(n)$ in the range of 0 255 . When fed to a D/A, this gave control voltages of $0-10 \mathrm{~V}$ which yielded electrode voltages ranging from $0-20 \mathrm{kV}$ from the high-voltage controller units.

The object was a metal-coated glass sphere, weighed $0.15 \mathrm{~g}$, and had a diameter of about $1 \mathrm{~cm}$. The camera distance was adjusted so that the object's image was 20 pixels in diameter. The gain parameters could be varied $\pm 15 \%$ and still yield stable levitation. However, there are interactions. For example, if $P G$ or $D G$ are both increased, oscillation of the sample is likely, especially after a large perturbation, indicating that the system is under-damped if the gains are too large. The stability of levitation is limited by the camera resolution to \pm 1 pixel. The other components of the system such as the $\mathrm{D} / \mathrm{A}$ converters are not limiting factors.

\section{A. Aing levitator}

Unfortunately, the situation in zero-g is not quite so simple. The electrode arrangement of Fig. 1, for example, would tend to toss the object right out of the chamber whenever the direction of field reversed, since it would then produce diverging lateral forces. This problem was resolved in the ring levitator by using two additional electrodes to control lateral centering. These electrodes, labeled 2 and 4 in Fig. 4, are kept at a higher positive potential relative to their respective central electrodes, 1 and 3, to cause a positive object to experience a force towards the center line if it drifts off center. In order to exert a force in the $+y$ direction, electrodes 1 and 2 are made more positive than 3 and 4 , respectively, whereas a force along $-y$ is obtained by making 3 and 4 more positive. The PID formula of Eq.(3) is still used for control along the axial dimension, but the PID output, $O(n)$ is now used to determine the electrode voltage by means of the transfer functions of Fig. 8. Thus, a PID output of 128 , corresponding to a zero axial force, places $+10 \mathrm{kV}$ on both electrodes 1 and 3 , and $\pm 13.5 \mathrm{kV}$ on 2 and 4 ; whereas a PID output of 200 would make the lower electrodes much more positive than the upper ones, corresponding to a force of approximately $100 \mathrm{dyn}$ in the $+y$ direction. Lateral centering force is lowered at the extremes of the PID output range in order to provide a maximum axial force without any sudden discontinuities.

Levitation occurs in $1 \mathrm{~g}$ with a $0.15 \mathrm{-g}$ sample at about $14-\mathrm{kV}$ voltage difference between the upper and lower plates. Thus, the levitation occurs near a PID output value of 128. In Fig. 8, this corresponds to the diamond-shaped region where the electrode transfer functions cross near the center of the diagram. Figure 6 shows a typical display during levitation in 1-g using the ring positioner described above. The values of the setpoint $S P$ (labeled SET in Fig. 6), $P G, I G, D G$, and $S V G$, and $M I D$ (MP, midpoint) are shown, as well as the values of the velocity and integral. The sample is at 29 , the commanded setpoint.

The actual voltages in kilovolts on the plates can be found by dividing each plate value by 255 and multiplying by $20 \mathrm{kV}$ giving: $12.6,15.4,7.4$, and $9.3 \mathrm{kV}$ on plates 1 to 4 , respectively. At the instant illustrated, there was a $5.2-\mathrm{kV}$ voltage difference between electrodes 1 and 3, giving an upward force on the positively charged sample. The top trace

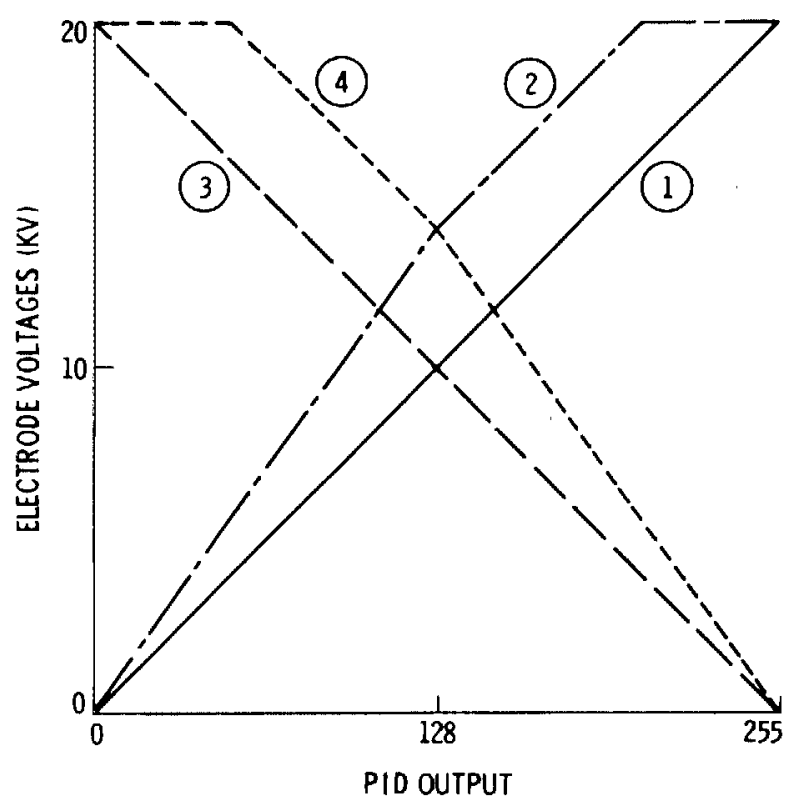

FIG. 8. Electrode voltages vs PID output transfer functions for the ring positioner. 
shows the position of the sample versus time for a period of $2.13 \mathrm{~s}$ while the lower trace shows the values for plate 1 in the same period. Note the recovery of the positioner after the camera image was interrupted for eight frames. The oscillation of the sample's position shows the servo to be underdamped in this case.

\section{B. Tetrahedral levitator}

The control algorithm for the tetrahedral positioner is complicated not only by the fact that it must maintain active control in all three spatial dimensions rather than just one, but also by the conceptual problem of converting from the $X-Y-Z$ Cartesian orientation of the cameras' position readings to the tetrahedral orientation of the electrodes. Several approaches to the problem, including position-dependent table look-up of electrode voltages for $X-Y-Z$ force vectors, were simulated on a computer. The algorithm eventually chosen for the actual positioner is based on the idea that, independent of other factors, the primary effect of a voltage on a given electrode is to exert a force on the charged sample pointing towards, or away from, that electrode. It, therefore, might be expected that if a desired $X-Y-Z$ setpoint was specified in terms of distances from each of the four electrodes, each electrode acting independently to maintain the sample at the specified distance from itself might, in fact, hold the sample at the desired position. The simulations bore out this idea.

The present servosystem is designed for objects with negative charge and the electrodes always have positive voltages. This choice is arbitrary. Simple modifications to the program could be made so that the servo would work with objects of either charge. The sample is negatively charged during levitation by making the top electrodes positive while the bottom electrode remains at ground potential at the time of launch. The object could be positively charged merely by reversing the potential on the electrodes.

The algorithm as implemented may be described as follows for positioning a negatively charged sample in zero-g. During each frame period the computer goes through the following steps to produce appropriate output signals:

(1) Read $X-Y-Z$ sample position from camera interface.

(2) Using known $X-Y-Z$ locations of each of the four electrodes compute the distances $d_{i}(n)$ where $i=1,2,3,4$ from the sample to each electrode.

(3) Use the PID algorithm to compute an output value for each electrode as follows:

$$
\begin{aligned}
0_{i}(n)= & M P+P G \cdot E(n)+I G \sum_{j=0}^{n}\left[d_{i}(j)-S P_{i}\right] \\
& +D G\left[d_{i}(n)-d_{i}(n-1)\right] \\
& +S V G\left[d_{i}(n)-d_{i}(n-4)\right],
\end{aligned}
$$

where

$$
\begin{aligned}
& M P=\text { midpoint(zero in the } 0-g \text { case), } \\
& I G=\text { integral gain, } \\
& D G=\text { derivative gain, } \\
& S V G=\text { slow velocity gain, } \\
& S P_{i}=\text { setpoint for electrode } i,
\end{aligned}
$$

$d_{i}(n)=$ distance from sample to electrode $i$ at the $n$th frame time.

(4) Find the smallest of the $0_{i}$, and subtract this value from all four $0_{i}^{\prime} s$ to yield values $P_{i}$. It should be noted that, as the implementation restricts the $0_{i}$ to an 8 -bit positive integer, the $P_{i}$ are greater than or equal to zero, and less than 256.

(5) Output the $P_{i}$ values to the $\mathrm{D} / \mathrm{A}$ converters resulting in a voltage of

$$
P_{i} \frac{2 \times 10^{4}}{255} \mathrm{~V}
$$

being placed on the $i$ th electrode.

Step (4) in the above procedure was included to help minimize the overall electrode voltages in order to reduce the probability of electrical discharge. It is a reasonable step because the field in the interior of the tetrahedral chamber is determined largely by the voltage differences between the electrodes. Also note that the MP term is not needed in $0-g$ conditions where no large force is needed to counter gravity. The position errors that arise are adequately compensated by the integral term.

In concluding this discussion of control algorithms, it is observed that the positioners we discussed here work, and work well for launching and holding solid samples of $0.15 \mathrm{~g}$ in $1-g$ and up to $3 \mathrm{~g}$ in $10^{-2}-\mathrm{g}$. As the sampling rate of such a control system increases with respect to the rates at which the physical system can change, it becomes less and less important to correctly predict how the system is going to respond to a change in a control output. Thus, a very simple control law can be adequate for controlling a complex nonlinear time-varying system without a detailed understanding of its physical dynamics. Successfully determining the parameters of the control algorithm may entail much testing. In this case, a simplified computer simulation of the system behavior was used to determine initial parameters and stability. In the limit, all that is necessary is that the control outputs change in the direction required to evoke the desired response more often than they change in the wrong direction. While we are nowhere near this limit, our levitation systems do fall in the former category, in which it is not necessary for the control system to accurately model or predict the behavior of the physical system.

\section{SORTWARE SYSTEM}

In addition to executing the control algorithm, the positioner system's computer is programmed to provide a number of other features. These include generating sample charging and launching sequences (see Sec. VI); interactive control of servosystem parameters; display of the system's status; and time keeping. The sample's position at each frame time, accelerometer data, and all servo parameters are recorded on floppy disk for subsequent detailed analysis. This data has been used, for example, to simulate the operation of the servo off line to study the servo's response to $g$ perturbations. The software is structured so that all functions such as data acquisition, servo control, and launch sequencing, which need to operate immediately upon the availability of sample coordinate information, are performed 
in a so-called foreground mode upon activation by a hardware interrupt signal from the camera interface. The interrupt signal is generated at the end of each frame at the rate of $120 \mathrm{~Hz}$, and also supplies the basic time interval for the realtime clock. Remaining tasks which may operate in a less time-critical manner are executed in background mode; that is, whenever control of the processor is relinquished by the foreground functions. These background routines share the remaining processor time on an equal priority basis, being activated in a round-robin fashion and executing until they either complete their function (such as the update of a status display), or are momentarily suspended for lack of further information (as in the input processing for control parameter changes from the keyboard when all keystrokes for the new value have not yet been typed), or by occurrence of a hardware interrupt from the camera interface activating calculation of the next servo output values.

Because of the demanding real-time nature of many of the above functions, and to the limited processing power of the 8-bit 6502 microprocessor used in our Apple II + control computer, all software for the levitator was implemented in ASSEMBL Y language (i.e., the native machine language of the computer) in order to assure maximum efficiency and speed. The foreground routines, consisting primarily of the servo control procedure, require approximately $10 \%$ of the total available processor time in the ring levitator, whereas they consume fully $70 \%$ of the available time in the tetrahedral levitator. The bulk of this $70 \%$ is spent performing multiplications and square root extractions in software.

The basic structure of the program is illustrated in Fig. 9, which illustrates the main servo program, MAIN executed in background mode, and Fig. 10, illustrating the interrupt request program (IRQ), executed each time the CCD camera begins a new frame and issues a hardware interrupt request. The main servo program contains variables, data structures, and linkage tables as well as tables of squares and

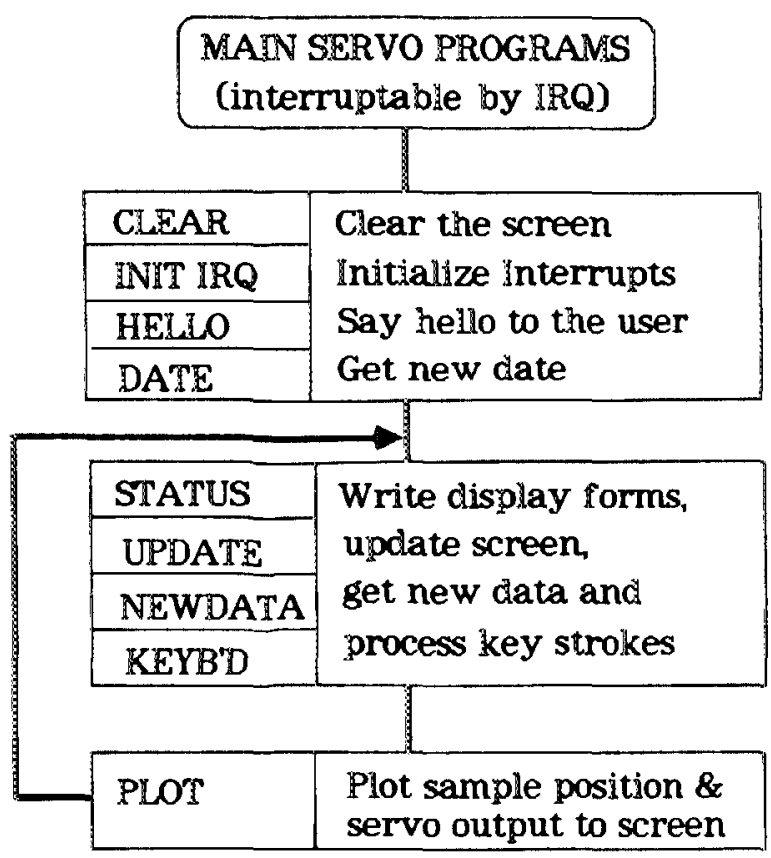

FIG. 9. Main servo program schematic.

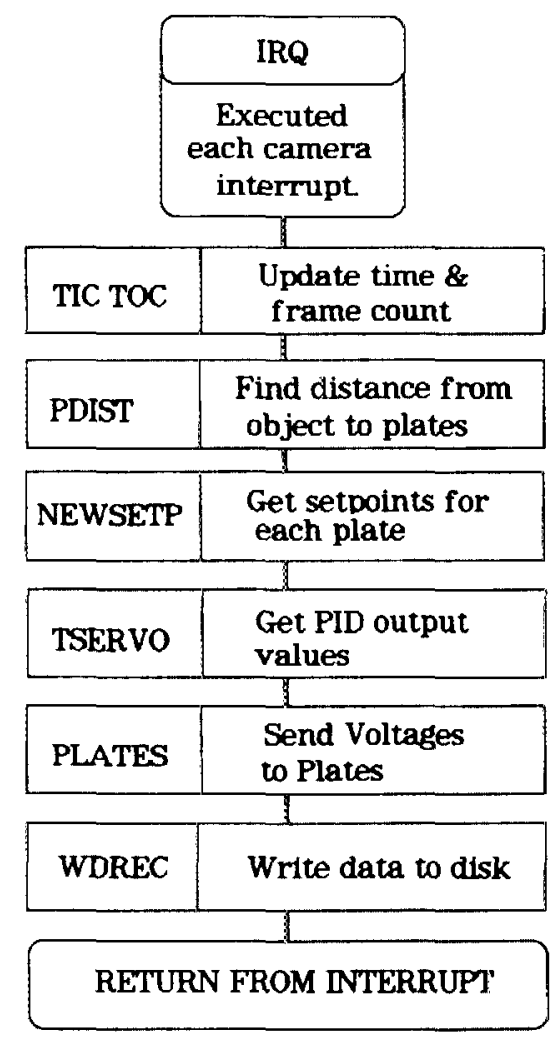

FiG. 10. IRQ-interrupt request program schematic.

square roots for coordinate calculations. MAIN initializes by clearing the screen; enabling interrupts; and setting initial servo gains, setpoints, various internal values, and the date.

MAIN then enters an endless loop which repeatedly executes STATUS to write display forms; UPDATE to write current data to the screen, as well as PLOT to draw the ball position and plate outputs and other data to the graphics screen from data read from memory by NEWDATA.

Inputs to MAIN are from the keyboard, by which the user types in new parameters, and controls the servo state. Typed characters are handled by keyboard service (KEY'BD). For example, typing " $H$ " turns on the high voltage and " $L$ " initiates the launch of a sample and turns on the servo. MAIN controls IRQ by writing values of various switches and data into memory. IRQ communicates with MAIN by writing object positions and servo variables into memory. These values are also displayed on the Apple's graphics page by STATUS and UPDATE. All the routines in MAIN are interruptable by IRQ.

MAIN is interrupted, and IRQ is activated each frame by a nonmaskable interrupt issued to the Apple bus by the camera interface. TIC TOC then updates the time and frame counters and reads the $\mathrm{A} / \mathrm{D}$ to acquire accelerations or other data. PDIST calculates the distance of the object from each plate by converting camera coordinates to plate coordinates using square and square root tables. NEWSETP then determines the current setpoints for each plate. This data is then used by TSERVO, the main servo program, which implements the PID algorithm described above by executing four times each interrupt, once for each plate.

Finally, PLATES converts the PID outputs to D/A 


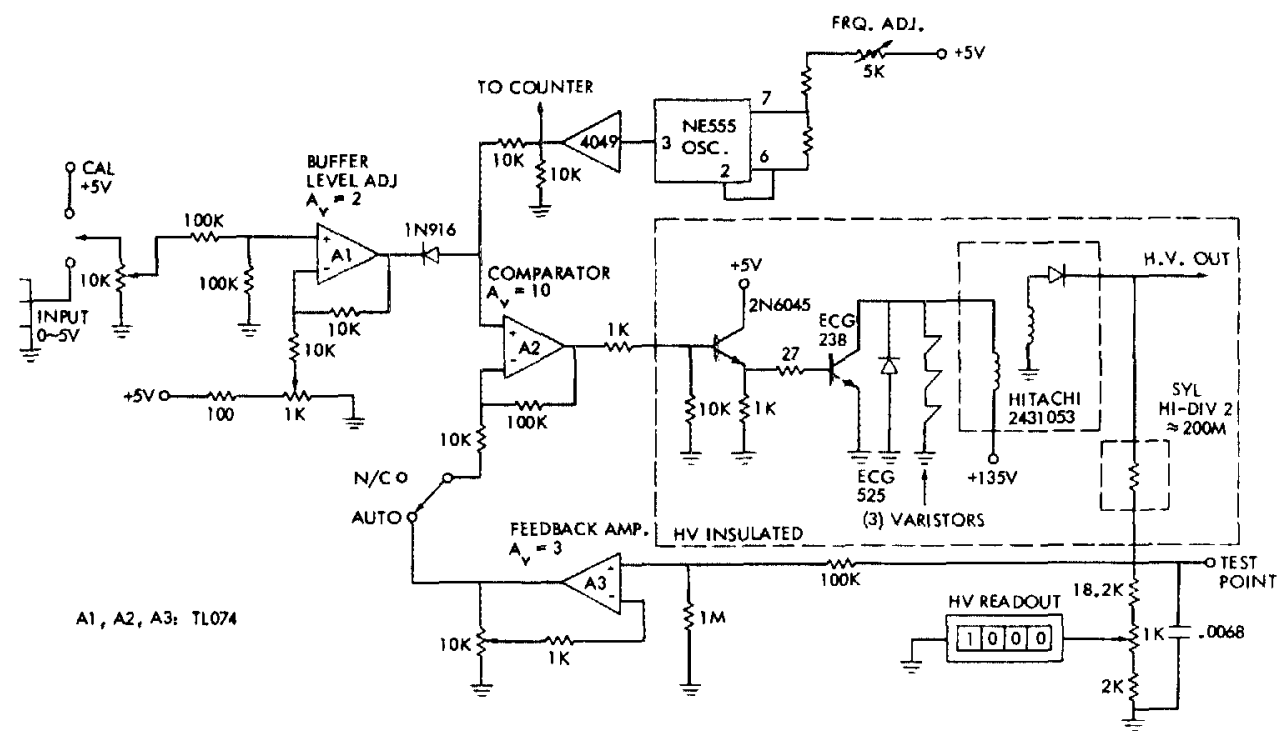

Fig. 11. A simplified schematic of a typical high-voltage unit.

commands which control the voltages on the plates. PLATES also contains subroutines that launch the object on the user's command. WDREC writes the object's position, acceleration, and the servo state to floppy disk once for each execution of IRQ.

Inputs to IRQ are the memory addresses where servo parameters from MAIN are stored, as well as object positions placed in memory by the camera interface through direct memory access (DMA) and accelerations and other data from an A/D. Except for the DMA of the camera interface which occurs with each camera frame, IRQ has the highest priority.

\section{HIGH-VOLTAGE UNITS}

The high-voltage units used in all positioners are designed around flyback transformers used in Hitachi color TV sets. In the TV sets, these transformers produce and maintain a constant $25-\mathrm{kV}$ dc. However, in our application the output high voltage can be changed by varying the amplitude of the driving input voltage. Among the advantages of utilizing flyback transformers for the electrostatic positioners are: (1) dynamic range is from 0 to $25 \mathrm{kV}(200 \mu \mathrm{A})$ with good linearity; (2) they have a fast response time $(\sim 1 \mathrm{~ms})$; and (3) they are compact, lightweight, and have no moving parts. Figure 11 shows a simplified schematic diagram of a high-voltage unit. The dashed box which includes a Hitachi flyback transformer is wired into a separate high-voltage protected box.

The frequency of a square wave generated by the NEC 555 oscillator is adjusted for the peak high-voltage output. The flyback transformers usually resonate at around 55 $\mathrm{kHz}$. The amplitude of the square wave is controlled by a bias voltage across a clamping diode. The servo input voltage, after going through a buffer amplifier and a level adjuster, eventually appears as a bias voltage across a clamping diode which in turn controls the amplitude of this square wave. This amplitude controlled square wave, when combined with the voltage stabilizing feedback signal at the comparator, is fed to the primary winding of the flyback transformer. The output of the transformer was half-wave rectified and filtered to obtain positive dc voltage with approximately $1 \% 55-\mathrm{kHz}$ ripple on it. Figure 12 shows a linearity curve of a typical high-voltage unit. Although it shows slight nonlinearity below $1.5 \mathrm{kV}$, this did not effect control significantly (see comments at the end of Sec. III).

\section{CHARGING AND LAUNCHING}

\section{A. Solid objects}

The charging and launching of solid objects in a 1-g environment is rather straightforward. When the ring levitator is used, the object is initially positioned in the middle of the lower electrode. Then a coordinate for the object's final desired position, a setpoint ( $\mathrm{SP}$ ) is typed into the computer. When a launch is initiated by typing an "L", the high-voltage units are turned on, a large voltage difference appears between the top and bottom electrodes. Since the object is in contact with the bottom electrode, it carries a large induced

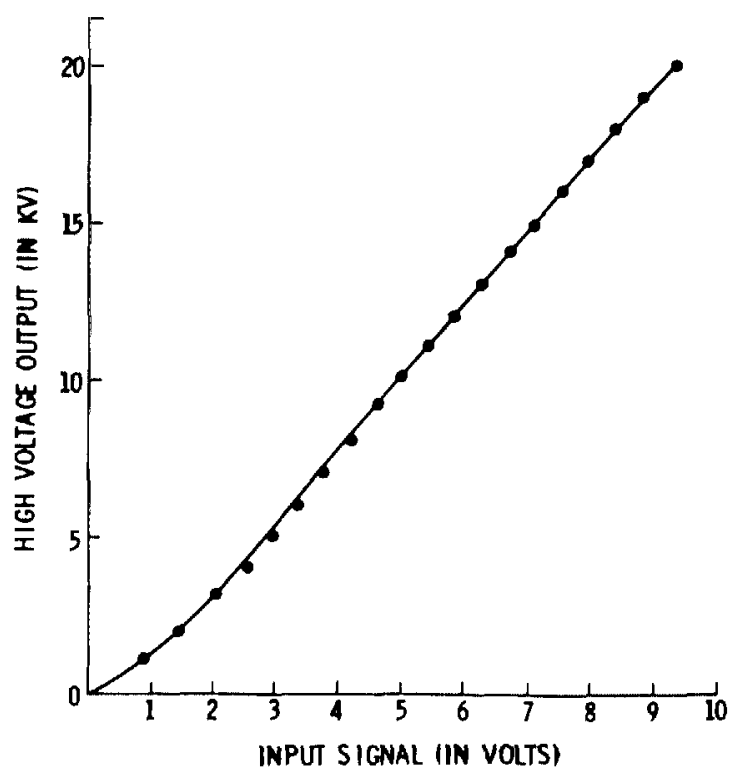

FIG. 12. A linearity curve of a typical high-voltage unit. 
charge and hops toward the preset position. As soon as the object's initial position changes, the servo program [Eqs. (3) and (4)] is activated. With properly set servo parameters, the object will be positioned in the middle, between the electrodes, after some transient oscillation subsides. At this stage one can start optimizing servo parameters. In fact, this process can be effectively carried out by observing the plot of ball position versus time created by briefly interrupting the field of view of a CCD camera. This causes a pulse in the ball position followed by a damped oscillation as the ball returns to the setpoint (see Fig. 6).

In the reduced $g$ environment, the object was initially held at the lower electrode with the help of a vacuum chuck. With the high voltage on, a sudden release of the vacuum frees the object which is repelled toward the setpoint. Knowing the weight of the ball and the voltage required to suspend it in $1 \mathrm{~g}$, we estimate the amount of charge on the object (a metallized sphere of $\sim 1.2 \mathrm{~cm}$ in diameter) is approximately $5 \times 10^{-9} \mathrm{C}$. If the vacuum chuck is not used to hold the object firmly in contact with the electrode prior to the launching, the object tends to leave the electrode prematurely before a large enough charge is accumulated on the object. This can be especially serious in the reduced $g$ environment because it produces unpredictable charge values, and the force applied by a given electrode voltage is then uncertain.

\section{Líquid drops}

Levitation of large ( $\approx 1-\mathrm{cm}$ diameter) liquid drops cannot be realized in a $1-g$ environment unless the liquid's surface tension is extremely high. For example, water drops larger than a few $\mathrm{mm}$ in diameter cannot be levitated in a $1-\mathrm{g}$ environment. Therefore, our liquid experiments were carried out using water in the reduced $g$ environment provided by the NASA KC-135 aircraft. The KC- 135 low gravity aircraft flies a series of parabolic (Keplerian) trajectories (Fig. 13), each of which yields approximately $25 \mathrm{~s}$ of low gravity environment $\left(\Delta g=10^{-2} g\right)$.

Launching a liquid drop is similar to launching solid objects in reduced gravity. The only difference is that the vacuum chuck is replaced by a retractable liquid injection nozzle with a small piece of nonwetting cloth (usually velvet)

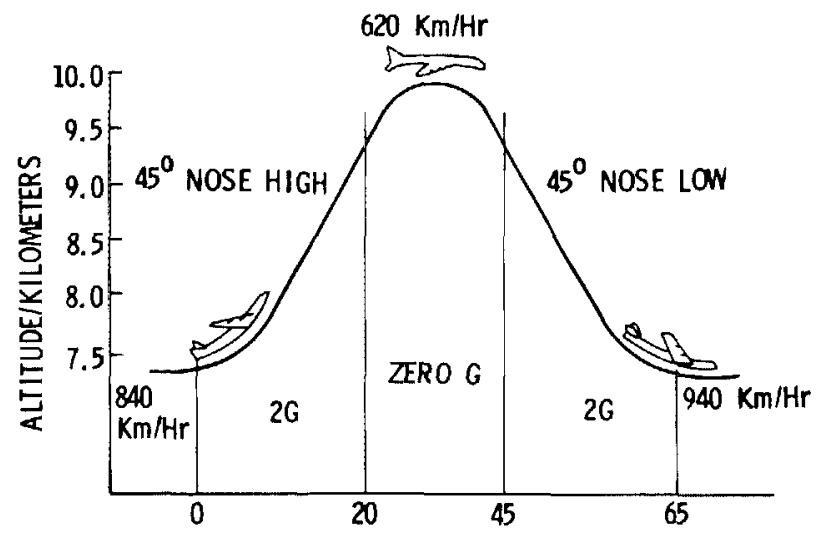

ZERO GRAVITY MANEUVER (SECONDS)

FIG. 13. A typical parabolic trajectory of the NASA KC-135 low gravity aircraft.

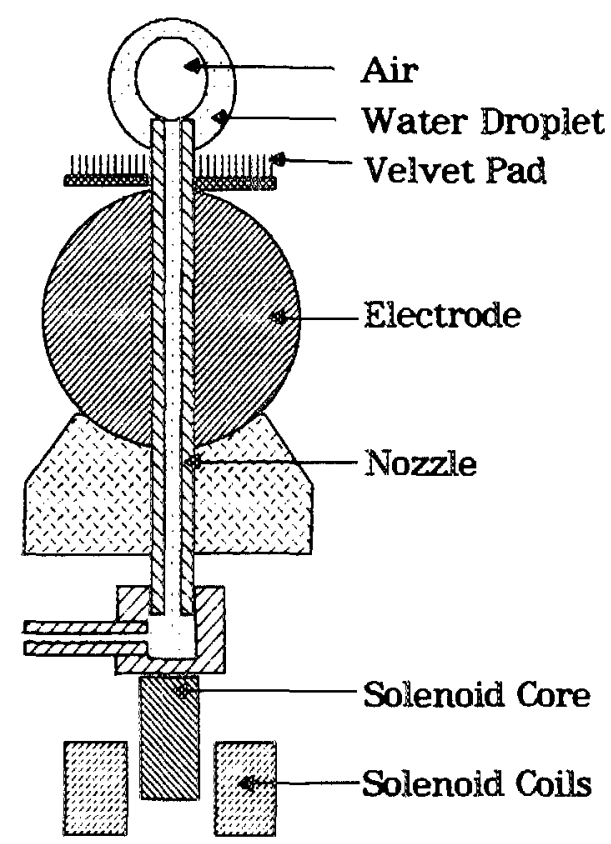

Fig. 14. Diagram of the liquid drop launching apparatus.

around the nozzle, as shown in Fig. 14. As soon as the zero-g portion of the parabola starts, a known amount of liquid is injected through the nozzle until a drop is fully formed at its tip. During this period, the drop is gently pulled toward the top electrodes by applying $+7 \mathrm{kV}$ on each of the top three electrodes while the bottom electrode and the nozzle were at ground potential. This pull prevented the drop from falling away from or breaking connection with the nozzle due to small perturbations of $g$. The velvet pad limited the wetting area of the liquid drop to the tip of the nozzle. When the launch key was pressed, the drop was fully charged by suddenly increasing the electrode voltage to nearly $+20 \mathrm{kV}$. At the same time, the nozzle was quickly retracted by a solenoid. The increased repulsion and retraction of the nozzle broke the charged drop free. The drop then moved toward its present position under control of the servo. If the drop is made out of conducting liquid, the induced charge is carried by the drop. If the liquid is nonconducting, a special charging method would have to be used. ${ }^{14}$

For the deployment of compound drops (a drop composed of a liquid shell surrounding a gas core) a predetermined amount of liquid-air-liquid column was injected sequentially through a nozzle. Again, the size of the wetting area around the nozzle has to be adjusted for the successful deployment and launch of compound drops. Figure 15 shows a few frames from a video camera taken during a successful zero- $g$ launching of a compound drop using the tetrahedral positioner. It shows one of the first successful levitations of a compound liquid drop in reduced gravity. (a) A dyed water drop with an air core is attached to the nozzle and resting on a velvet pad, ready to be launched. (b) It is repelled from the nozzle with the application of voltage under servo control. (c) The neck is being broken showing a Taylor cone. (d) The compound drop is in the air and being held at the preset position. 

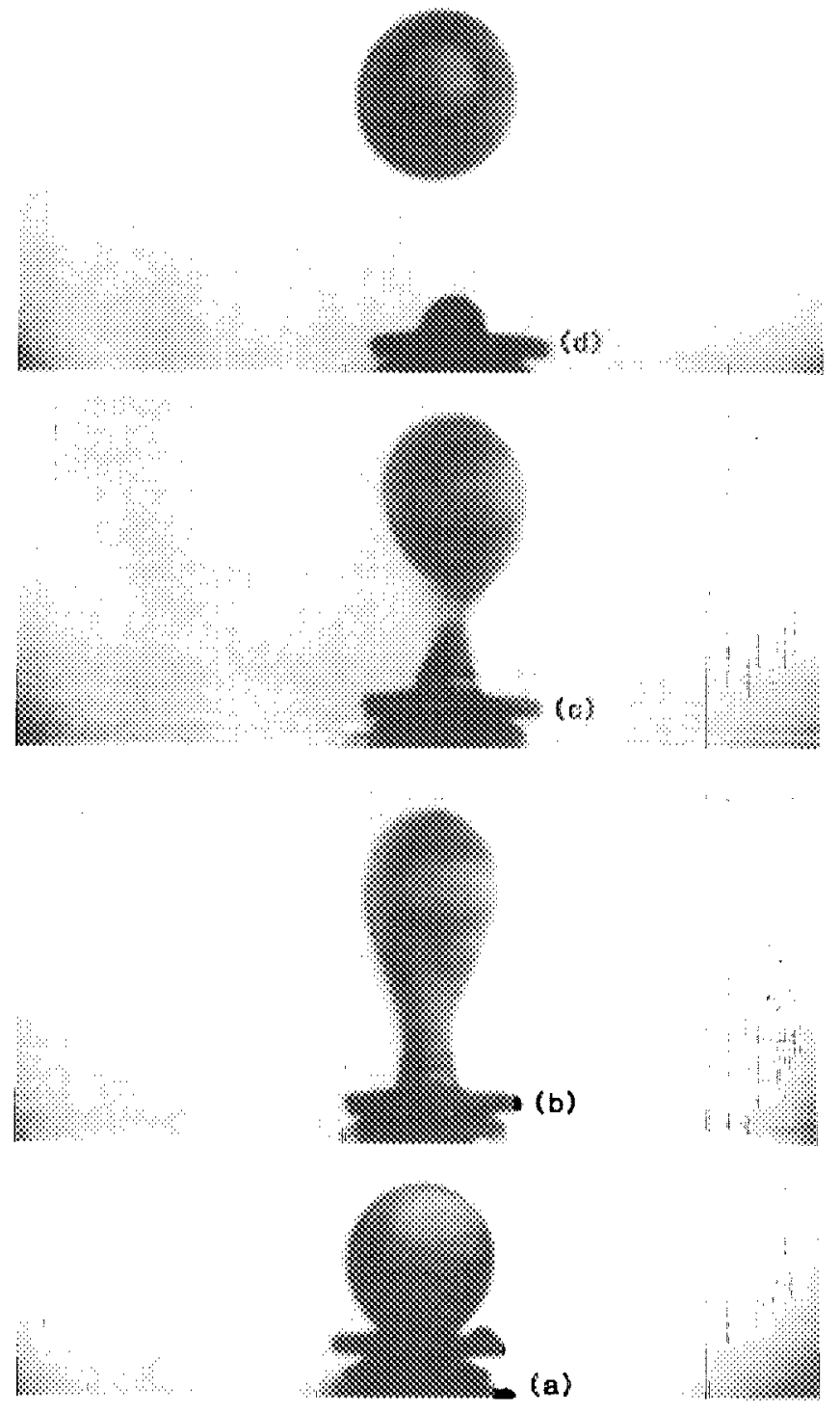

FIG. 15. A typical launch of a liquid drop. The drop shown here is a compound drop with an outer water shell and an inner air core.

\section{DISCUSSION}

In a high $g$ environment, a large portion of the available electric force has to be dedicated to counterbalance the object's weight, i.e., for levitation rather than positioning and controlling. At our current maximum allowable voltage of $20 \mathrm{kV}$, the maximum allowable weight is approximately 0.15 $\mathrm{g}$ in the $\mathrm{l} g$ environment. Some of the solid objects we have levitated in $1-g$ are shown in Fig. 16. These objects range from silver-painted styrofoam to metallized hollow glass shells.

In the reduced $g$ environment provided by the KC-135, the mass of the objects can be substantially increased since all the control voltage can be used to compensate small $g$ perturbations instead of levitating against the large constant $g$ force. Assuming a maximum $g$ perturbation of $3 \times 10^{-2} g$ within the $\mathrm{KC}-135$, the maximum mass which our present

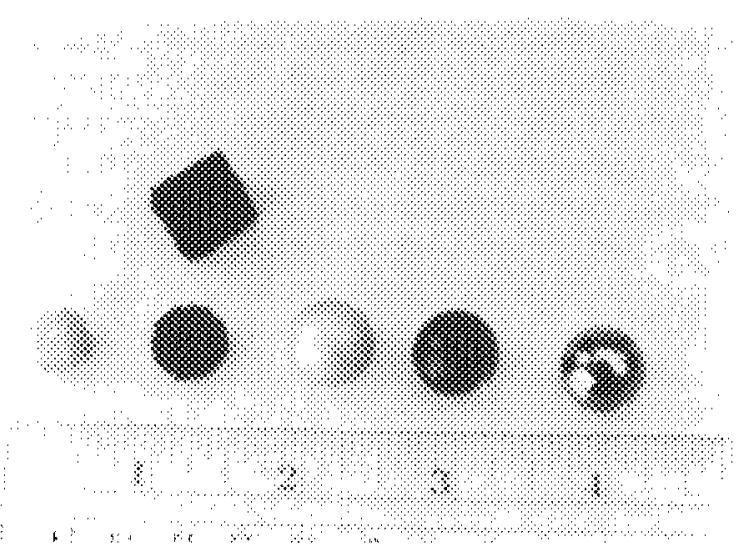

Fig. 16. Some solid objects that have been levitated in a $1-g$ environment From the left, the objects are: silver-painted Styrofoam, carbon impregnated conductive foam pieces, silver-painted glass shell, ink-coated glass shell, and platinum-coated glass shell.

electrostatic positioner can handle is approximately $5 \mathrm{~g}$. We have, in fact, held 3-g objects on the KC-135. This maximum limit can easily be extended to over $500 \mathrm{~g}$ since within the space shuttle the $g$ perturbation would be significantly reduced $\left(\approx 10^{-4} \mathrm{~g}\right)$.

Though this paper summarizes preliminary results for electrostatic positioners, other important issues, such as charging methods for nonconducting materials, charge gain or loss at high temperatures, and techniques for charged drop oscillation and rotation, etc., are under investigation.

\section{ACKNOWLEDGMENTS}

We gratefully acknowledge Dr. Melvin Saffren for his contribution in the initial stage of this program. We would like to thank D. Migliori and D. Barber for their technical assistance together with S. Thomas and G. Yepez for their editorial assistance. The work described in this paper was carried out by the Jet Propulsion Laboratory, California Institute of Technology, under contract with the National Aeronautics and Space Administration.

\footnotetext{
'In the Space Shuttle, typical $g$ perturbations are caused by the astronauts movements $\left(10^{-1} \sim 10^{-4} \mathrm{~g}\right)$, atmospheric drag $\left(\sim 5 \times 10^{-6} \mathrm{~g}\right)$, or thruster firings $\left(10^{-3} \sim 10^{-4} \mathrm{~g}\right)$.

${ }^{2}$ For Earnshaw's theorem, see S. A. Stratton, Electromagnetic Theory (McGraw-Hill, New York, 1941).p. 116.

${ }^{3}$ There is "Electrodynamic containment of charged particles" which does not require feedback control for levitation, however, this technique is limited to small particles. See, R. F. Wuerker, H. Shelton, and R. V. Langmuir, J. Appl. Phys. 30, 342 (1959).

${ }^{4}$ T. G. Wang, M. M. Saffren, and D. D. Elleman, AIAA paper no. 74-155, 1974.

${ }^{5}$ C. H. Allen and I. Radnick, J. Acoust. Soc. Am. 19, 857 (1947).

"Alan E. Crawford, Ultrasonic Engineering (Butterworth, London, 1960).

${ }^{7}$ R. F. Bunshah, Techniques of Metals Research, edited by R. F. Bunshah (Interscience, New York, 1968), Vol. 1, Part 2, Chap. 18.

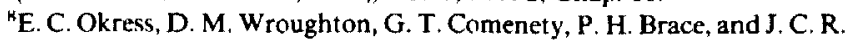
Kelly, J. Appl. Phys. 23, 545 (1952).

${ }^{9}$ E. Fromm and H. Jehn, Br. J. Appl. Phys. 653 (1963).

${ }^{10} \mathrm{G}$. Wouch, R. T. Frost, N. P. Pinto, G. H. Keith, and A. E. Lord, Jr.,
} Nature 274, 235 (1978). 
${ }^{11}$ W. K. Rhim, M. Saffren, and D. D. Elleman, Proceedings of Material Processing in Reduced Gravity Environment of Space, edited by G. E. Rindone, pp. 115-119, 1982.

${ }^{12}$ P. F. Clancy, E. G. Lierka, R. Grossbach, and W. M. Heide, Thirtieth Congress, International Astronautical Federation, Munich, Federal Re- public of Germany, 17-22 September 1979.

${ }^{13}$ J. A. Cadzow and H. R. Martnes, Discrete Time and Computer Control Systems (Prentice Hall, Englewood, NJ, 1970).

${ }^{14} \mathrm{C}$. D. Hendricks, Electrostatics and Its Applications, edited by A. D. Moore (Wiley, New York, 1973), p. 53. 